\title{
The early intervention of geniposide and PNS combination modulated $A \beta$ production and synaptic plasticity in young APP/PS1 transgenic mice
}

\section{Yan Tan}

Beijing University of Chinese Medicine

Fang He ( $\nabla$ hefangbucm2014@163.com )

Beijing University of Chinese Medicine https://orcid.org/0000-0002-5530-4576

Jiao Li

Beijing University of Chinese Medicine

Yali Zhang

Beijing University of Chinese Medicine

Liushu Yi

Beijing University of Chinese Medicine

Ce Zhang

Beijing University of Chinese Medicine

\section{Ruoxi Gu}

Beijing University of Chinese Medicine

Ke Yang

Beijing University of Chinese Medicine

Jiani Zhang

Beijing University of Chinese Medicine

Peng Wei

Beijing University of Chinese Medicine

\section{Xu Wang}

Beijing University of Chinese Medicine

Qian Hua

Beijing University of Chinese Medicine

\section{Research}

Keywords: A $\beta$, Alzheimer's disease, Geniposide, PNS, synaptic plasticity

Posted Date: April 21st, 2020

DOI: https://doi.org/10.21203/rs.3.rs-22530/v1 
License: (c) (i) This work is licensed under a Creative Commons Attribution 4.0 International License. Read Full License

Version of Record: A version of this preprint was published at Journal of Alzheimer's Disease \& Parkinsonism on January 1st, 2018. See the published version at https://doi.org/10.4172/21610460.1000448 . 


\section{Abstract \\ Background}

Alzheimer's disease (AD) is a common neurodegenerative disease with age-related, which accounts for nearly $80 \%$ of dementia. Accompanied with aging population worldwide and clinical failures in AD drugs, early prevention and diagnosis are under main concern. Traditional Chinese medicine (TCM) has a long history in the prevention of cognition declines. In clinic, the combination of geniposide and Panax notoginseng saponin (PNS) is clinically efficacious in the treatment of ischemic cerebral stroke and vascular dementia. In vivo, under a preventive strategy, the combination can improve spatial learning and memory and reduce amyloid plaques in a variety of AD-like animal models.

\section{Methods}

In order to illustrate the neuroprotective role of geniposide and PNS combination under the early intervention strategy, we medically administrated at one-month age of APP/PS1 transgenic mice for three months. The morphology of pyramidal neurons in hippocampus CA1 was observed after Hematoxylin and Eosin staining (HE staining) and the dendritic spines in hippocampus CA1 area are by Golgi silver staining. Thereafter we detect the level of $A \beta 1-40$ and $A \beta 1-42$ in hippocampus and in cortex by ELISA. In addition, western blotting was used for further understanding the effects of geniposide and PNS combination in APP/PS1 transgenic mice.

\section{Results}

We found that the ratio of $A \beta_{1-42} / A \beta_{1-40}$ in both cortex and hippocampus was significantly decreased under the treatment; secondly, the combination significantly increased dendrite spine density in hippocampus CA1 areas, and increased the protein level of PSD-95 (synaptic function-related protein in post-synapse).

\section{Conclusion}

These results indicated that geniposide and PNS combination modulated A $\beta$ production and synaptic plasticity in the early stage of $A D$ processing.

\section{Background}

Traditional Chinese Medicine (TCM) has experienced more than 2000 years in oriental countries, followed by a general principle referring to that compatible components of different herbal decoction may jointly play a role in synergistically enhance curative efficacy or reduce adverse effects. In the theory of TCM, the pathogenesis of dementia has been considered as the damage of brain collateral by toxins. Combined 
with the knowledge of AD pathological changes, inflammation, amyloid plaques could be the toxins in TCM, which resulted in the damage of synaptic plasticity and connections, and eventually leaded to cognition declines. Based on the theory, the formulae prescribed according to detoxification and collateral-dredging strategy has been administrated in dementia, such as the combination of geniposide (GP) and Panax notoginseng saponin (PNS). In clinic, the combination has been used in ischemic stroke patients and also whose memory declined [1]. GP is the main bioactive ingredient of Fructus Gardeniae, which is considered as a function of clearing away heat and toxic materials, also is regarded as diminishing inflammation in modern medicine [2,3]. PNS is the main bioactive ingredient of Panax notoginseng, which is considered as activating blood circulation to dissipate stasis $[4,5]$. In vivo, the combination improved spatial learning and memory and recued amyloid plaques in several AD-like animal models $[1,6,7,8]$; in vitro, GP and PNS showed to protect neuronal damage caused by formaldehyde $[9,10]$ and promoted the neurite outgrowth of rat hippocampal neurons under oxygen/glucose-deprivation conditions [11]. These data suggest that GP and PNS combination undergoes a potential role of neuroprotection in AD.

In this study, due to further investigate the neuroprotective effect of GP and PNS, in vivo, we administrated at one-month age of APP/PS1 Tg mice, lasting for three months. Firstly, we focused on analyzing the production of $A \beta$ in both cortex and hippocampus; secondly, we detected the plasticity of synapses, such as the number of dendritic spines in hippocampus CA1 areas and the expression level of synaptic related protein.

\section{Materials And Methods}

\section{Animals}

APP/PS1 transgenic mice were purchased from Nanjing Biomedical Research Institute of Nanjing University (Cat. D000268), and bred in independent cages. APP/PS1 mice were randomly divided into five groups, including transgenic model (Tg), Aricept, PNS, GP and GP+PNS group, and one littermate wild type control (WT) group. Each group contained fifteen mice. The animal housing rooms were maintained under standard laboratory conditions, $12 \mathrm{~h} / 12 \mathrm{~h}$ light-dark cycle, $23 \pm 1^{\circ} \mathrm{C}$ air temperature, and $55 \pm 15 \%$ humidity, with access to water and food. All procedures concerning care, treatment and dissection are in accordance with the regulations of ethical committee for use of experimental animals of the Chinese Academy of Sciences.

\section{Drugs}

GP and PNS were purchased from Nanjing Ze-Lang Pharmaceutical Company (Cat. 20110502, Cat. ZL20110620GNPG), administrated with $0.01927 \mathrm{mg} / \mathrm{g} /$ day and $0.01668 \mathrm{mg} / \mathrm{g} /$ day, respectively, according to our previous studies; Aricept was brought from Eisai China Inc. (Cat. 070624A), treated with $0.0000714 \mathrm{mg} / \mathrm{g} /$ day (6). Drugs were dissolved in physiological saline and mixed in powder food to form a drug ball. Before the drug feeding, the mice were fasted for 2-3h. Treatments were administrated at onemonth age and lasting for three months. 


\section{Hematoxylin and Eosin Staining}

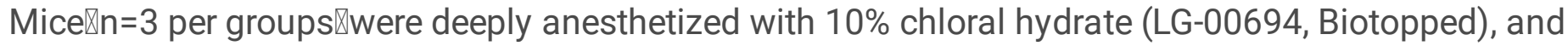
perfused intracardially with ice-cold phosphate-buffered saline (PBS), followed by ice-cold $4 \%$ paraformaldehyde (P6148, Sigma) in 0.1M PBS. Brains were quickly removed from the skull and postfixed in $4 \%$ paraformaldehyde overnight at $4^{\circ} \mathrm{C}$. Brain samples were processed in paraffin embedding on the following day. Coronal brain sections were performed serially at $6 \mu \mathrm{m}$-thick of each slide. Staining was performed as follows: 1) glass slides with paraffin sections were put in to a hot air oven at $60^{\circ} \mathrm{C}$ for $1 \mathrm{~h} ; 2$ ) process deparaffinization as following steps, each step last for 3min: Xylenes, new Xylenes, 50\% Xylenes/50\% ethanol, $100 \%$ ethanol, new $100 \%$ ethanol, $95 \%$ ethanol, $70 \%$ ethanol, $50 \%$ ethanol, distilled water; 3) dip rehydrated slides $30 \mathrm{sec}$ in Hematoxylin, then rinse with tap water until water runs clear, further dip slides $2 \mathrm{~min}$ in Eosin Y, then rinse with tap water until water runs clear; 4) run slides through a quick dehydration series, 3 quick dips in each of the following solutions: $50 \%$ ethanol, $70 \%$ ethanol, $95 \%$ ethanol, $100 \%$ ethanol, new $100 \%$ ethanol, then incubate slides $1 \mathrm{~min}$ in $50 \%$ Xylenes $/ 50 \%$ ethanol, $3 \mathrm{~min}$ in Xylenes, 3 min in new Xylenes. Eventually, mount with neutral balsam sealed by cover glass. Sections were observed and photographed under the microscope (Nikon).

\section{Golgi Staining}

The animals ( $n=4$ per groups) were deeply anesthetized with 10\% chloral hydrate (LG-00694, Biotopped). Monitor them until the point when the animal fails to respond to pinching of the foot. The brain tissue was prepared followed by Hito Golgi-Cox OptimStain Kit (Catalog number: HTKNS1125, Hitobiotec Inc.). Briefly, remove the brain tissue and rinse it in double-distilled water for 2-3 seconds to remove blood from the surface. Transfer tissue in the impregnation solution and store at room temperature in the dark. Replace the impregnation solution after 18 hours, and store at room temperature for fourteen days in the dark. Transfer tissue into Solution-3, and store at $4^{\circ} \mathrm{C}$ in the dark. Replace Solution-3 after 12 hours, and continue to store at $4^{\circ} \mathrm{C}$ in the dark for 72 hours. Freeze tissue in freezing isopentane (on dry ice) for 30 seconds to 1 minute. Remove the frozen tissue and store at $-70^{\circ} \mathrm{C}$. Prepare cryostat sections at $80 \mu \mathrm{m}$ thickness. Air dry slides. Rinse slides in distilled water for 3 minutes, twice. Stain slices for 10 minutes in staining solution. Rinse slides in distilled water for 4 minutes, twice. Dehydrate slides followed by $50 \%$, $75 \%$ and $95 \%$ ethanol for 5 minute each. Dehydrate slides in 100\% ethanol for 5 minutes, three times. Clear in xylene for 5 minutes, twice. Apply coverslip over sections using mounting medium. Dry and observe under a 100x oil-immersion objective lens (OLYPUS). Pyramidal cells in hippocampus CA1 area are selected, analyzing its apical and basal spine density in dendritic branch at the length of more than $10 \mu \mathrm{m}$. Data is quantified with Image $(1.48 \mathrm{v})$.

\section{Western Blotting}

Brain lysates $(n=4)$ were prepared according to previous experiment. Briefly, hippocampus and cortex were separately homogenized by a mixture of RIPA (Cat. CW2336, CWBIO), PMSF (Cat. 0754-5G, Amresco) and phosphatase inhibitor cocktails as 100:1:1 ratio. The brain homogenate solutions were centrifuged at $15000 \mathrm{rpm}$ for $30 \mathrm{~min}$ at $4^{\circ} \mathrm{C}$. Then collect the supernatant for Western blot assay. Protein 
concentration was measured by protein assays (Cat. 23225, Pierce) following manufacturer's instructions. Protein samples were electrophoresed through $12 \%$ SDS-polyacrylamide gels and transferred to Polyvinylidene Fluoride Membrane. Membranes were blocked with 5\% skim milk in PBST for $1 \mathrm{~h}$ at room temperature, then incubated with primary antibodies at $4^{\circ} \mathrm{C}$ overnight with shaking at $60 \mathrm{rpm} / \mathrm{min}$. Primary antibodies used are: rabbit anti-PSD95 polyclonal antibody (Cat. P78352, Cell signaling) at 1:1000, mouse anti $\beta$-actin monoclonal antibody (Cat. CW0096, Cwbio) at 1:2000. On the following day, wash with PBST for $5 \mathrm{~min}, 3$ times. Then, incubate with secondary antibody for $1 \mathrm{~h}$ at room temperature. Secondary antibodies used are: HRP-conjugated goat anti-rabbit (Cat. CW0103, Cwbio) at 1:5000 or goat anti-mouse (Cat. CW0102, Cwbio) at 1:5000 or rabbit anti-goat (Cat. E030130-01, Earthox) at 1:10000. Then, repeat washing step. Protein signals were detected with enhanced chemiluminescence reagent (Cat. 32109, Pierce), and exposed with X-ray film in dark room.

\section{Enzyme-linked Immunosorbent Assay}

Insoluble $A \beta_{1-40}$ and $A \beta_{1-42}$ were analyzed with enzyme-linked immunosorbent assay. Briefly, tissues (hippocampus or cortex, $n=5$ ) were dissolved in $98 \%$ of formic acid, split for 15 minutes on ice, then centrifuged at $17000 \mathrm{rpm}$ for $1 \mathrm{~h}$ at $4{ }^{\circ} \mathrm{C}$. Collect the supernatants for later measurement, using $A \beta_{1-40}$ and $A \beta_{1-42}$ ELISA kit (Cat. KHB3482 and KHB3442, Invitrogen-Biosource). Measurement was normalized according to the manufacturer's instructions.

\section{Statistical}

Data was analyzed by One-way ANOVA to compute statistical significance in each group compared with $\mathrm{Tg}$ group. All data were presented as mean \pm standard error of the mean (SEM) and $p<0.05$ was considered statistically significant. Analyses were carried out using SPSS20.0.

\section{Results}

The morphology of pyramidal neurons in hippocampus CA1 area was abnormal in APP/PS1 mice, while it was improved under the early intervention of GP and PNS combination.

Under an early intervention, GP, PNS and their combination has been administrated at one-month age of APP/PS1 transgenic mice, followed by 3-month long (Fig. 1A). Firstly, by Hematoxylin and Eosin staining (HE staining), the morphology of pyramidal neurons in hippocampus CA1 area was detected. At fourmonth age, a severe condensed but uneven and thinner layer of pyramidal neurons was observed in APP/PS1 mice, which was different from WT mice. Under all the intervention groups, a relative thicker and smoother but without severe condensed layer of pyramidal neurons has been found, which was similar to WT group (Fig. 1B, shown in double-headed arrows). These observations indicated that the abnormal morphology of pyramidal neurons in hippocampus CA1 area occurred as early as four-month age of APP/PS1 mice, while GP and PNS intervention can reverse the abnormality. 
The ratios of $A \beta 1-42 / A \beta 1-40$ in both hippocampus and cortex were evidently increased in APP/PS1 mice, while GP and PNS combination significantly reduced the ratios but adjusted the production of $A \beta$ differently in hippocampus and cortex.

APP/PS1 mouse is one of the most widely used AD-like animal, since it develops amyloid plaques in brain by six to seven months of age [12], accompanied with an accumulating production of $A \beta$ since three to four months of age [13]. At four-month age, we found that the ratios of $A \beta_{1-42} / A \beta_{1-40}$ in both hippocampus and cortex were significantly increased in APP/PS1 mice, while only the group of GP and PNS combination significantly reduced the rations (Fig. 2A-B). Furthermore, in order to illustrate the changes of $A \beta_{1-42}$ and $A \beta_{1-40}$ production in different brain areas, the levels of insoluble $A \beta_{1-42}$ and $A \beta_{1-40}$ were measured. Interestingly, in the hippocampus area, the production of $A \beta_{1-42}$ was significantly reduced compared with APP/PS1 mice; while in the cortex area, the production of $A \beta_{1-40}$ was significantly increased compared with APP/PS1 mice (Fig. 2C-D). But in positive control group, Aricept did not show a significant change in their ratios or insoluble $A \beta$ productions. These data indicated that GP and PNS combination showed a different pattern of $A \beta$ production in different brain tissues, suggesting that different mechanisms of $A \beta$ clearance may exist in different areas.

In Aricept group, compared with Tg mice, there is a reduction tendency observed in the production of both $A \beta_{1-42}$ and $A \beta_{1-40}$, but we did not find a significant change, as well as the ratio of $A \beta_{1-42} / A \beta_{1-40}$ did not decrease. This data showed that at four-month age of APP/PS1 Tg mice, Aricept did not reverse the A $\beta$ production.

\section{The number of dendritic spines was significantly in hippocampus CA1 areas of APP/PS1 mice, while GP and PNS combination significantly increased the number.}

Accumulated $A \beta$ peptides have been considered as a toxicant to neurons, especially in hippocampus related with learning and memory [14]. In vivo, we narrowed to hippocampus CA1 area to detect the synapse plasticity. Golgi silver staining has been used to label dendrites and quantify both apical and basal dendritic spines in hippocampus CA1 areas. At 4-month age, the dendritic spines at hippocampus CA1 area in WT mice are evenly dispersed with a high density. However, unlikely to WT, in Tg mice, their dendritic spines occurred sporadically uneven with low density, suggesting an abnormal morphology of synapse in Tg at the age of four-month. In addition, under a three-month treatment of TLJN (PNS + GP), similar to WT, it shows a high density and evenly dispersed. But in Aricept, we did not observe a significant improvement in the density of dendritic spines (Fig. 3A).

Due to statistic analyze the number of dendritic spines in hippocampus CA1 area, its number has been counted in both apical and basal ends. Compared with WT, the number of dendrite spines in both apical and basal ends is significantly decreased in Tg mice. After treatment, in term of apical dendrites, GP and PNS + GP groups significantly increase the number of dendrite spines compared with $\mathrm{Tg}$ (Fig. 3B); in terms of basal dendrites, PNS, GP and PNS + GP groups significantly increase the number of their dendrite spines compared with $\mathrm{Tg}$ (Fig. 3C). However, Aricept did not significantly increase the number of 
dendritic spines in both apical and basal ends. These results suggest that PNS, GP, PNS + GP can reverse the impaired morphology of synapses in terms of increasing both apical and basal dendrites spines, while Aricept has no evident effect on increasing dendritic spines number.

\section{TLJN (PNS + GP) increases the expression level of postsynaptic density protein 95 (PSD-95) in APP/PS1 hippocampus.}

Synaptic disruption accompanies with AD pathogenesis; its impairment of synaptic plasticity indicates memory deficits, but occurred earlier than behavior symptoms $[15,16]$. Postsynaptic density protein 95 (PSD-95), a major scaffolding protein locating at excitatory synapses anchors synaptic proteins, regulates synaptic distribution, plays an important role in synaptic plasticity. Since the abnormal morphology of synapses observed in APP/PS1 Tg mice at four-month age, here, we further evaluate the protein level of PSD-95 (Fig. 4). Compared with WT mice, the protein level of PSD-95 is significantly decreased in Tg mice. Under the treatment of PNS + GP, PSD-95 protein level is significantly increased compared with Tg. This data is consistent with Golgi silver staining in PNS + GP group with a higher dendritic spines (Fig. 3), indicating that TLJN (PNS + GP) can improve synaptic plasticity by accelerating the expression level of postsynaptic related protein PSD-95 and dendritic spines. However, unfortunately, we found that Aricept further decreased the protein level of PSD-95 compared with Tg, suggesting that Aricept did not enhance the plasticity of synapses at four-month age of APP/PS1 Tg mice (Fig. 4).

\section{Discussion}

Alzheimer disease (AD) is a progressive neurodegenerative disease, accounting for $50-75 \%$ of all dementia patients worldwide (http://www.alz.co.uk/). However, their diagnoses are often missed or delayed due to the lower prevalence of dementia in younger people and large variability in etiologies [17, 18].. And, non-effective drugs against $A D$ in market approved accumulate the serious situation, which puts the prevention and treatment into an impending place [19]. Then, early this year, FDA proposed that the drug development and diagnosis of $A D$ should be focused on the early stage before the onset of overt dementia [20].

TCM has a long history in oriental countries. Preventing Disease, an essential consideration of TCM treatment strategy, emphasizes the conception of prevention and early treatment of diseases. Based on this thinking, for the past ten years, we have been tried to find and explain the neuroprotective role of TCM in AD. According to previous studies, in vitro, we found that GP and PNS combination alleviated the damage of ischemia/reperfusion neurons by promoting the secretion of active substances from brain microvascular endothelial cells (BMECs) [21]; under oxygen/glucose-deprivation condition, the combination protected both primary rat hippocampal neurons and BMECs from cell death [11]; again, under formaldehyde stress, TLJN inhibited the cell apoptosis by modulating the expression of $B C l-2, P 53$, caspase 3 and caspase 9 , and increasing the activity of intracellular superoxide dismutase and glutathione peroxidase $(9,10)$. These data suggest that it can prevent neurons from cell death, but also can protect BMECs. In vivo, to further verify its efficacy on learning and memory, we used a rat model and 
three other different AD Tg mice. In rat, after $A \beta_{25-35}$ injection into the bilateral hippocampus $C A 1$ areas, we found that GP and PNS prolonged a cavity delitescence, decreased arm entries in Y-maze test, and reduced amyloid plaques in rat brain by up-regulating insulin-degrading enzyme and neprilysin levels [1]; in APP/V717I Tg mice, GP and PNS improved their spatial memory deficits in both Water-Maze test and inhibitory avoidance test (Step-down test) at ten-month age [6]; in APP/PS1 Tg mice, we found that it improved the spatial learning and memory in Water-Maze test and reduced amyloid plaques in brain at 10-month age (data have not published yet); in APP23 Tg mice, we observed an inhibitory effect of the combination on amyloidogenic APP processing by down-regulating the cleavage enzymes BACE1 at twelve-month age [8]. These evidence showed that GP and PNS can improve learning and memory in different $A D \mathrm{Tg}$ mice, and reduce the deposition of amyloid plaques.

Based on these studies, we continued to investigate its neuroprotective role in an early stage. We used APP/PS1 Tg mice. These mice express human APP (695 Swedish mutation) and a mutant human presenilin1 (PS1-dE9). Both mutations are associated with early-onset AD. They develop beta-amyloid deposits in brain by six to seven months of age [12], but their memory deficits usually occurred at the age of nine-month and older [22]. APP/PS1 Tg mouse is one of the most widely used AD-like animal, due to the extensive $A \beta$ deposition and synaptic deficit with aging, accompanied with memory deficits. At 4months old of APP/PS1 Tg mice, there is an approximately 30\% reduction in spine density as well as about $20 \%$ decrease in both dendritic area and dendritic diameter in the transgenic mice as compared with wild-type littermates [23]. In addition, A $\beta$ accumulation in APP mutant neurons reduces PSD-95 and AMPA receptor (GluR1) in synapses [24]. Then, by using young APP/PS1 Tg mice, we focused on the prevention started from one-month age, lasting for four-month age, which is before formation of amyloid deposition.

At the early stage, we firstly detected the production of $A B$. Both $A \beta_{1-42}$ and $A \beta_{1-40}$ are significantly increased in APP/PS1 Tg mice at four-month age. The ratio of $A \beta_{1-42} / A \beta_{1-40}$ is significantly increased as well compared with WT group. Secondly, to detect its morphology in hippocampus CA1 areas, we found the pyramidal cell layer is severe condensed with uneven thickness in Tg group compared with WT mice. in addition, according to clinical studies, the loss of synapses has been considered as one of the most reliable index of cognition deficits in both postmortem and biopsied AD brain [24]; and amyloid deposition or $A \beta$ peptide (either insoluble $A \beta$ or soluble $A \beta$ species) is associated with synaptic abnormalities, such as dendritic spine loss [25], breakage of neuronal branches, spine dysfunction and collapse [26]. We further investigated the change of synaptic plasticity. We found that the number of dendritic spines is significantly reduced in Tg mice at four-month age; again, the protein level of PSD95, a synaptic-related protein, is significantly reduced; in hippocampus DG areas, the number of AMPA receptor positive cells is significantly reduced as well. These data suggest that APP/PS1 Tg mice exhibited a neurodegeneration processing at an early age (four-month age) before the formation of amyloid plaques and cognition impairment occurred. Then, under the early treatment, GP + PNS significantly decreased the ratio of $A \beta_{1-42} / A \beta_{1-40}$ ratio in both hippocampus and cortex. Interestingly, in hippocampus, the production of $A \beta_{1-42}$ was reduced under TLJN treatment; while in cortex, it was the production of $A \beta_{1-40}$ 
significantly increased. Secondly, we focused on the changes of synaptic plasticity. We found that the number of dendritic spines in hippocampus CA1 areas was significantly increased in GP + PNS group compared with Tg group. In addition, due to detect synaptic plasticity, we examined the expression level of synaptic-related protein, such as PSD-95. Our data showed that the combination has an impact on increasing the protein level of PSD-95. These data suggest that GP + PNS can maintain the delicate balance of $A \beta$ production in APP/PS1 Tg mice to reduce the ratio of $A \beta_{1-42} / A \beta_{1-40}$ in both hippocampus and cortex; and it can enhance synaptic plasticity at four-month age of APP/PS1 Tg mice (Fig. 5).

In this study, we also used Aricept as a positive control group, since it is the first drug approved by FDA to treat $A D$ in clinic. Aricept (also called donepezil), as a cholinesterase inhibitor, leads to a temporary slowdown in the loss of cognitive function by decreasing cholinesterase activity, resulting in higher acetylcholine (Ach) levels and improved brain function with mild-to-moderate AD patients [27, 28]. However, we did not observe evident effects of Aricept on APP/PS1 Tg mice at four-month age. Firstly, Aricept did not significantly reduce the ratio of $A \beta_{1-42} / A \beta_{1-40}$ in both hippocampus and cortex; secondly, in terms of synaptic plasticity, Aricept did not significantly increase the number of dendritic spines in hippocampus CA1 areas compared with Tg mice; and, the protein level of PDS-95 was further decreased compared with Tg mice. There might be there reasons: 1) Aricept is a cholinesterase inhibitor. In this study, we did not focus on the change of Ache level. But in terms of $A \beta$ production, at an early age of APP/PS1, before the formation of amyloid plaques, Aricept did not show an evident effect to maintain the balance of $A \beta$ production; 2 ) again, in terms of synaptic plasticity, before the cognition impairment occurred, Aricept did not show a positive role to enhance the health of synapses; 3 ) according to clinic studies, Aricept cannot stop the progressive loss of neurons or halt the progressive deterioration of cognitive faculties in AD patients [29]. This may provide us a hint that this limitation might be a hinder to its efficacy in an early stage. In addition, the side effects of Aricept should be matters, since it significantly increases ratings for vigour and anxiety symptoms [30].

To summary, preventing Disease is an essence strategy in TCM for treatments, especially in chronic disease and complicated diseases. Base on previous studies, in this experiment, we highlights the neuroprotective role of GP and PNS combination in an early stage. Before the formation of amyloid plaques, we found that GP and PNS can maintain the delicate balance of $A \beta$ production to reduce the ratio of $A \beta_{1-42} / A \beta_{1-40}$ in both hippocampus and cortex at four-month age of APP/PS1 Tg mice; before the cognition impairment occurred, the combination can enhance synaptic plasticity at an early age. These results suggest that the administration of GP and PNS in an early stage of APP/PS1 Tg mice can protect neuron damage from $A \beta$ accumulation and promote the health of synapses.

\section{Conclusions}

PNS + GP treatment starts at one-month age of APP/PS1 Tg mice lasting for three months, exhibits multiple functions in both cortex and hippocampus (Fig. 5). In cortex, PNS + GP can increase the production of $A \beta 1-40$; on the contrary, in hippocampus, PNS + GP decreases the production of $A \beta 1-42$. These two different effects on $A \beta$ production both leads to a reduction of $A \beta 1-42 / A \beta 1-40$ ratio in the 
end. Furthermore, PNS + GP can improve synaptic plasticity in hippocampus of APP/PS1 Tg mice. Both apical and basal dendritic spines are significantly increased under PNS + GP treatment. And post-synaptic related proteins PSD-95 and AMPA are higher expressed in PNS + GP group.

This study can provide important clues for the prevention of Alzheimer's disease, and also provide a theoretical basis for Chinese medicine as a preventive drug for difficult diseases. We expounded the vital material basis of traditional Chinese medicine as preventive and health medicine. It suggests that the combination of the two drugs may be an important means to prevent Alzheimer's disease.

\section{Abbreviations}

AD: Alzheimer's disease; A : Amyloid beta; TCM: Traditional Chinese medicine; GP: geniposide; PNS: Panax notoginseng saponin; HE: Hematoxylin and Eosin; PSD-95: Postsynaptic density protein 95; BMECs: brain microvascular endothelial cells.

\section{Declarations}

\section{Acknowledgements}

Not applicable.

\section{Authors' contributions}

Conceptualization, Y.T. and Q.H.; Performed the experiments, F.H. and Y.T.; writing-original draft preparation, Y.T. and J.L.; methodology and statistical analysis, J.L.,Y.L.-Z.,F.H.,L.S.-Y,C.Z.,R.X.-G,K.Y.,J.N.Z,P.W., and X.W.; writing-review and editing, Y.T. and Q.H.; supervision, Q.H.; project administration, Y.T. and Q.H.; funding acquisition, Y.T. and Q.H.; All authors have read and agreed to the published version of the manuscript.

\section{Funding}

The work was supported by grants from the National Natural Science Foundation of China Project (No. 81904049, 81973690) and Young Elite Scientists Sponsorship Program by CAST (Project No. CACM2018-QNRCC2-C06).

\section{Availability of data and materials}

The data used to support the findings of this study are available from the corresponding author upon request.

\section{Ethics approval and consent to participate}

All procedures in this study were approved and supervised by the animal research ethical committee of Beijing University of Chinese Medicine, and strictly obeyed the rules of animal experiment ethic to reduced 
number as well as suffering of animals.

\section{Consent for publication}

The manuscript is approved by all authors for publication.

\section{Competing interests}

The authors declare that they have no competing interests.

\section{Author details}

1 Beijing University of Chinese Medicine, No. 11 North 3rd Ring, Eastern Road, Beijing 100029, China. 2 Xi'an Satellite Control, Xi'an, China 710043

\section{Publisher's Note}

Springer Nature remains neutral with regard to jurisdictional claims in published maps and institutional afliations.

\section{References}

1. Liu Y, Hua Q, Lei H, Li P (2011) Effect of Tong Luo Jiu Nao on Abeta-degrading enzymes in AD rat brains. Journal of ethnopharmacology 137(2):1035-1046.

2. Cai L, Li CM, Tang WJ, Liu MM, Chen WN, Qiu YY, Li R (2018) Therapeutic Effect of Penta-acetyl Geniposide on Adjuvant-Induced Arthritis in Rats: Involvement of Inducing Synovial Apoptosis and Inhibiting NF-KB Signal Pathway. Inflammation doi: 10.1007/s10753-018-0861-0.

3. Che X, Wang M, Wang T, Fan H, Yang M, Wang W, Xu H (2016) Evaluation of the Antidepressant Activity, Hepatotoxicity and Blood Brain Barrier Permeability of Methyl Genipin. Molecules 21(7):E923.

4. Zheng M, Zhao M, Tang L, Zhang C, Song L, Wang W (2016) Ginsenoside Rg1 attenuates hypoxia and hypercapnia-induced vasoconstriction in isolated rat pulmonary arterial rings by reducing the expression of p38. Journal of thoracic disease 8(7):1513-1523.

5. He X, Deng FJ, Ge JW, Yan XX, Pan AH, Li ZY (2015) Effects of total saponins of Panax notoginseng on immature neuroblasts in the adult olfactory bulb following global cerebral ischemia/reperfusion. Neural regeneration research 10(9):1450-1456.

6. Yang K, Tan Y, Wang F, Zhang Q, Sun P, Zhang Y, Yao N, Zhao Y, Wang X, Fan A, Hua Q (2014) The improvement of spatial memory deficits in APP/V717I transgenic mice by chronic anti-stroke herb treatment. Experimental biology and medicine 239(8):1007-1017.

7. Zhang Y, Lin S, Hua Q, Yang K, Yao N, Yi L, Wang A, Chen W, Chen J (2012) Research on the mechanism of the active ingredients of Sanchi and Gardenia removing the early amyloid in the $A D$ transgenic mouse brain. Chinese Pharmacological Bulletin 28(2):173-179. 
8. He P, Li P, Hua Q, Liu Y, Staufenbiel M, Li R, Shen Y (2013) Chronic Administration of Anti-Stroke Herbal Medicine TongLuoJiuNao Reduces Amyloidogenic Processing of Amyloid Precursor Protein in a Mouse Model of Alzheimer's Disease. PloS one 8(3).

9. Ping Sun J-yC, Jiao Li, Meng-ru Sun (2013) The protective effect of geniposide on human neuroblastoma cells in the presence of formaldehyde. Complementary and Alternative Medicine 13:152-164.

10. Chen J, Sun M, Wang X, Lu J, Wei Y, Tan Y, Liu Y, Gotz J, He R, Hua Q (2014) The herbal compound geniposide rescues formaldehyde-induced apoptosis in N2a neuroblastoma cells. Science China Life Sciences 57(4):412-421.

11. Li XJ, Hou JC, Sun P, Li PT, He RQ, Liu Y, Zhao LY, Hua Q (2012) Neuroprotective effects of tongluojiunao in neurons exposed to oxygen and glucose deprivation. Journal of ethnopharmacology 141(3):927-933.

12. Reiserer RS, Harrison FE, Syverud DC, McDonald MP (2007) Impaired spatial learning in the APPSwe + PSEN1DeltaE9 bigenic mouse model of Alzheimer's disease. Genes, brain, and behavior 6(1):54-65.

13. He J, Qiao JP, Zhu S, Xue M, Chen W, Wang X, Tempier A, Huang Q, Kong J, Li XM (2013) Serum $\beta$ amyloid peptide levels spike in the early stage of Alzheimer-like plaque pathology in an APP/PS1 double transgenic mouse model. Current Alzheimer Research 10(9):979-86.

14. Selkoe DJ, Hardy J (2016) The amyloid hypothesis of Alzheimer's disease at 25 years. EMBO molecular medicine.

15. Yuki D, Sugiura Y, Zaima N, Akatsu H, Takei S, Yao I, Maesako M, Kinoshita A, Yamamoto T, Kon R, Sugiyama K, Setou M (2014) DHA-PC and PSD-95 decrease after loss of synaptophysin and before neuronal loss in patients with Alzheimer's disease. Scientific reports 4:7130.

16. Gruart A, Lopez-Ramos JC, Munoz MD, Delgado-Garcia JM (2008) Aged wild-type and APP, PS1, and APP + PS1 mice present similar deficits in associative learning and synaptic plasticity independent of amyloid load. Neurology of Disease 30(3):439-50.

17. Shichun Tu S-iO, Stuart A Lipton and Huaxi Xu (2014) Oligomeric Abeta-induced synaptic dysfunction in Alzheimer's disease. molecular neurodegeneration 9(48):12.

18. Cacace R, Sleegers K, Van Broeckhoven C (2016) Molecular genetics of early-onset Alzheimer's disease revisited. Alzheimer's \& dementia : the journal of the Alzheimer's Association 12(6):733-748.

19. Millenaar JK, Bakker C, Koopmans RT, Verhey FR, Kurz A, de Vugt ME (2016) The care needs and experiences with the use of services of people with young-onset dementia and their caregivers: $a$ systematic review. International journal of geriatric psychiatry.

20. FDA. U.S. Department of Health and Human Services FDA CfDEaR (2018) Early Alzheimer's Disease: Developing Drugs for Treatment Guidance for Industry. Draft guidance, 1-10.

21. Hua Q, Qing X, Li P, Li W, Hou J, Hu J, Hong Q, Sun P, Zhu X (2010) Brain microvascular endothelial cells mediate neuroprotective effects on ischemia/reperfusion neurons. Journal of ethnopharmacology 129(3):306-313. 
22. Leigh Holcomb MNG, Eileen McGowan, Xin Yu, Stan Benkovic, Paul Jantzen, Kristal Wright, Irene Saad, Ryan Mueller, Dave Morgan, Sunny Sanders, Cindy Zehr, Kassandra O'Campo, John Hardy, Cristian-Mihail Prada, Chris Eckman, Steve Younkin, Karen Hsiao, Karen Duff (1998) Accelerated Alzheimer-type phenotype in transgenic mice carrying both mutant amyloid precursor protein and presenilin 1 transgenes. Nature Medicine 4(1):4.

23. Webster SJ, Bachstetter AD, Nelson PT, Schmitt FA, Van Eldik LJ (2014) Using mice to model Alzheimer's dementia: an overview of the clinical disease and the preclinical behavioral changes in 10 mouse models. Frontiers in genetics 5:88.

24. Smith DL, Pozueta J, Gong B, Arancio O, Shelanski M (2009) Reversal of long-term dendritic spine alterations in Alzheimer disease models. Proceedings of the National Academy of Sciences of the United States of America 106(39):16877-16882.

25. Shankar GM, Walsh DM (2009) Alzheimer's disease: synaptic dysfunction and A 3 . Molecular Neurodeneration 4:48.

26. Culmone V, Migliore M (2012) Progressive effect of beta amyloid peptides accumulation on CA1 pyramidal neurons: a model study suggesting possible treatments. Frontiers in computational neuroscience 6:52.

27. Hardy CJD, Hwang YT, Bond RL, Marshall CR, Ridha BH, Crutch SJ, Rossor MN, Warren JD (2017) Donepezil enhances understanding of degraded speech in Alzheimer's disease. Ann Clin Transl Neurol 4(11):835-40.

28. Schneider LS (2014) Idalopirdine for Alzheimer's disease: written in the stars. The Lancet Neurology 13(11):1063-1065.

29. Wilkinson D, Windfeld K, Colding-Jørgensen E (2014) Safety and efficacy of idalopirdine, a 5-HT6 receptor antagonist, in patients with moderate Alzheimer's disease (LADDER): a randomised, doubleblind, placebo-controlled phase 2 trial. The Lancet Neurology 13(11):1092-1099.

30. Godyn J, Jonczyk J, Panek D, Malawska B (2016) Therapeutic strategies for Alzheimer's disease in clinical trials. Pharmacological reports : PR 68(1):127-138.

\section{Figures}




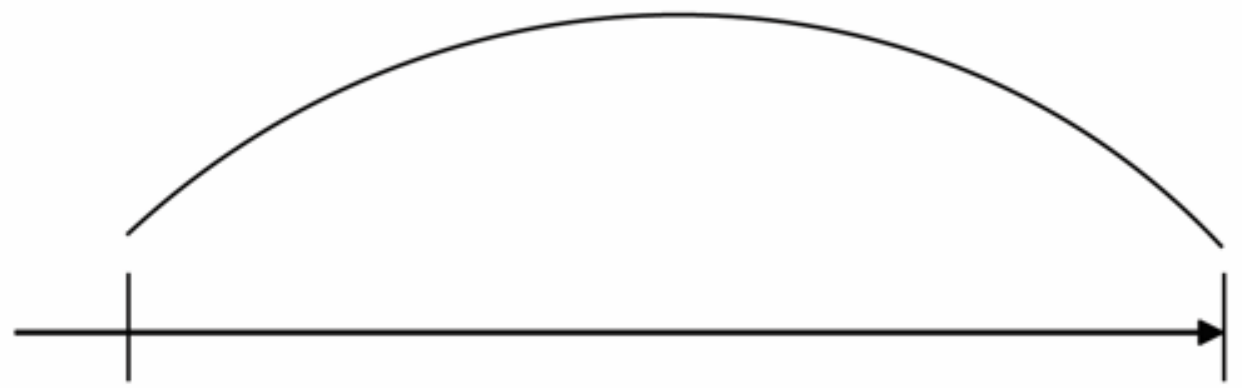

\section{One-Month age Grouping}

\section{Four-Month age Analyzing}
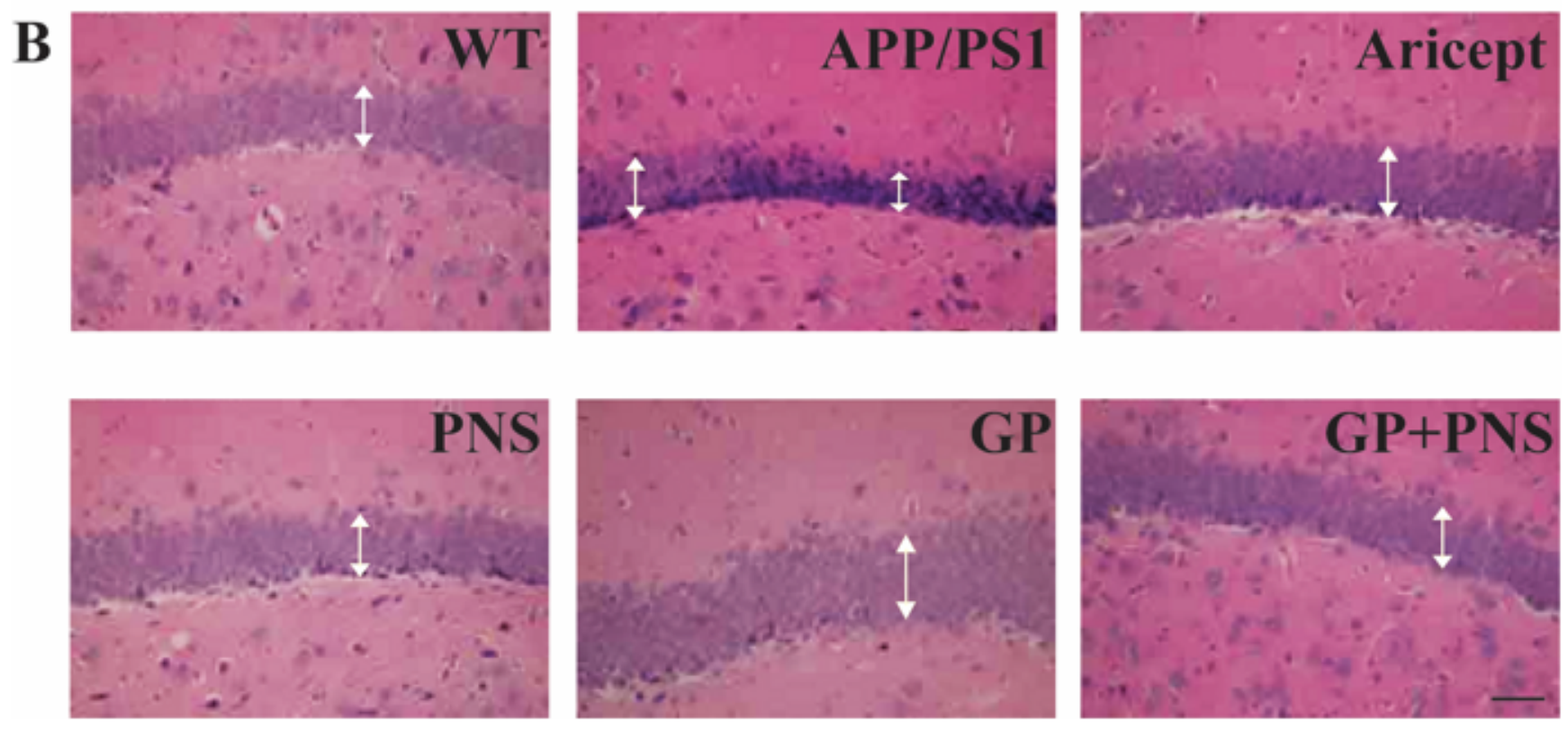

\section{Figure 1}

TLJN improves the abnormal morphology of pyramidal neurons in hippocampus CA1 aera occurred in APP/PS1 Tg mice. (A)The treatment strategy in APP/PS1 Tg mice. At one-month age, APP/PS1 Tg mice are randomly grouped into five groups: Tg, Aricept, PNS, GP and PNS+GP. Their wild-type littermates are considered as control without drug treatment. The drug administration lasts for three months. At fourmonth age, exert a series of analysis. (B).To exhibit the morphology of pyramidal neurons in hippocampus CA1 area, Hematoxylin and Eosin staining (HE staining) has been used. At 4-month old age, compared with wildtype group (WT), an severe condensed layer of pyramidal neurons occurred in APP/PS1 Tg mice (Tg). The thick of this layer is uneven; and, some part of it is strikingly thinner in $\mathrm{Tg}$ compared with WT (shown in double-headed arrow). In treatment groups (Aricept, PNS, GP and PNS+GP), the condensed layer consisting pyramidal neurons is evidently thicker and smoother than Tg group, which is similar to WT. Scale bar: $50 \mu \mathrm{m}$. 

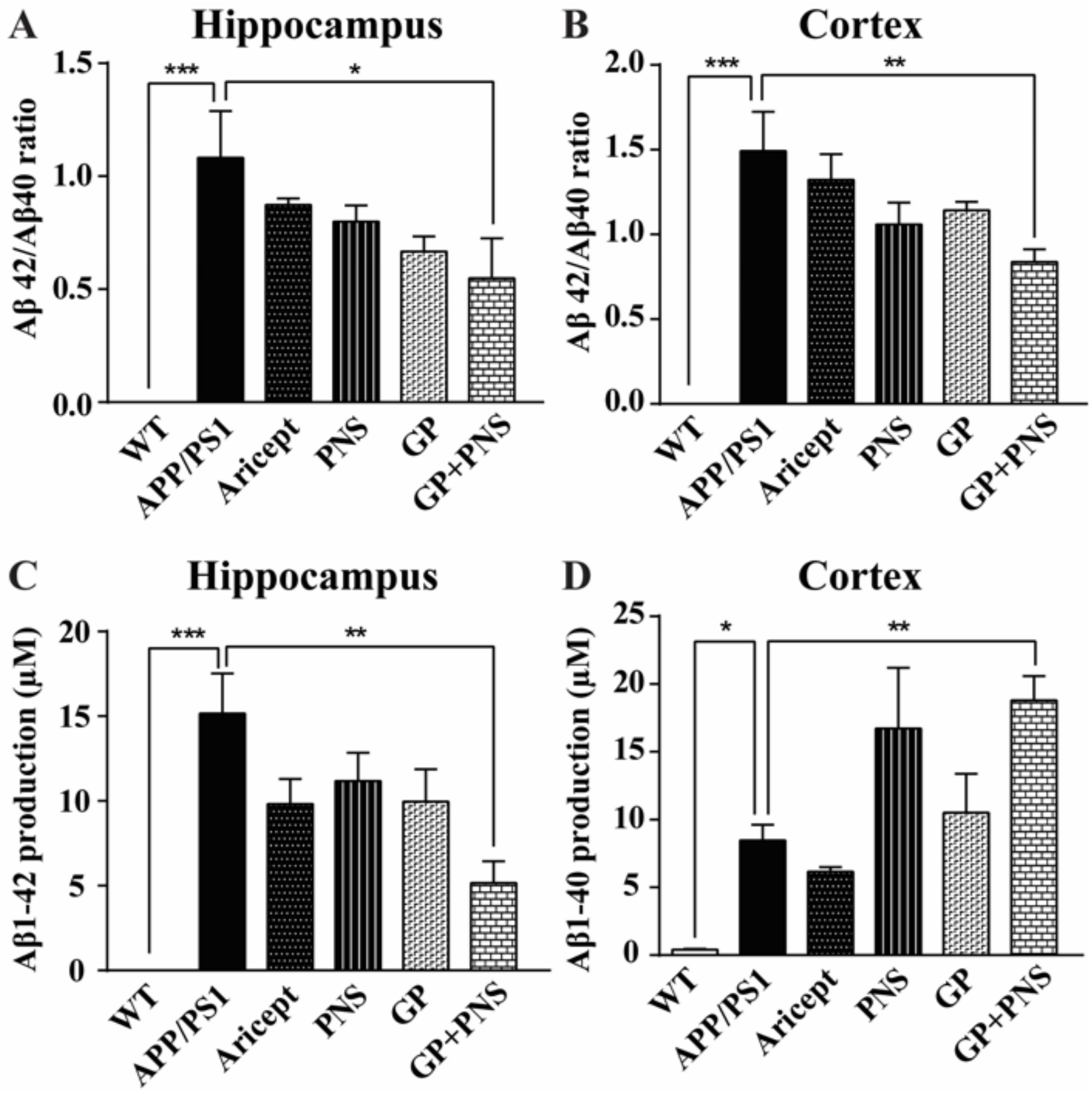

Figure 2

TLJN significantly reduces the ratio of $A \beta 1-42 / A \beta 1-40$ in both hippocampus and cortex, but adjusts the production of $A \beta$ differently. (A).Due to detect the level of $A \beta 1-40$ and $A \beta 1-42$, ELISA has been used. In hippocampus ( $n=5)$, we can't detect AB1-42 in WT mice at 4-month age. The level of AB1-42 in Tg mice is strikingly higher. Compared with $\mathrm{Tg}$ group, only PNS+GP significantly decreased the production of Aß142. Data are represented as mean \pm SEM. ${ }^{\star \star *} p<0.001$, ${ }^{\star *} p<0.01$. (B). In hippocampus $(n=5)$, there is a strikingly higher ratio of $A \beta 1-42 / A \beta 1-40$ occurred in Tg mice. Again, compared with Tg, only PNS+GP significantly reduced the ratio of $A \beta 1-42 / A \beta 1-40$. Data are represented as mean $\pm S E M$. *** $p<0.001$, * $p$ 
$<0.05$. (C). In cortex $(n=5)$, we can detect a slight amount of AB1-40 in WT mice at 4-month age. The level of A 1-40 in Tg mice is evidently higher. However, interestingly, compared with Tg group, PNS+GP significantly increases the production of $A \beta 1-40$ in cortex. Data are represented as mean \pm SEM. $* * p<$ $0.01, * p<0.05$. (D) Similar to hippocampus, in cortex $(n=5)$, there is a strikingly higher ratio of $A \beta 1-$ 42/AB1-40 occurred in Tg mice. What's more, we observe a significant reduction of AB1-42/AB1-40 ratio occurred in PNS+GP group. Data are represented as mean \pm SEM. ${ }^{\star \star \star} p<0.001, * \star p<0.01$.
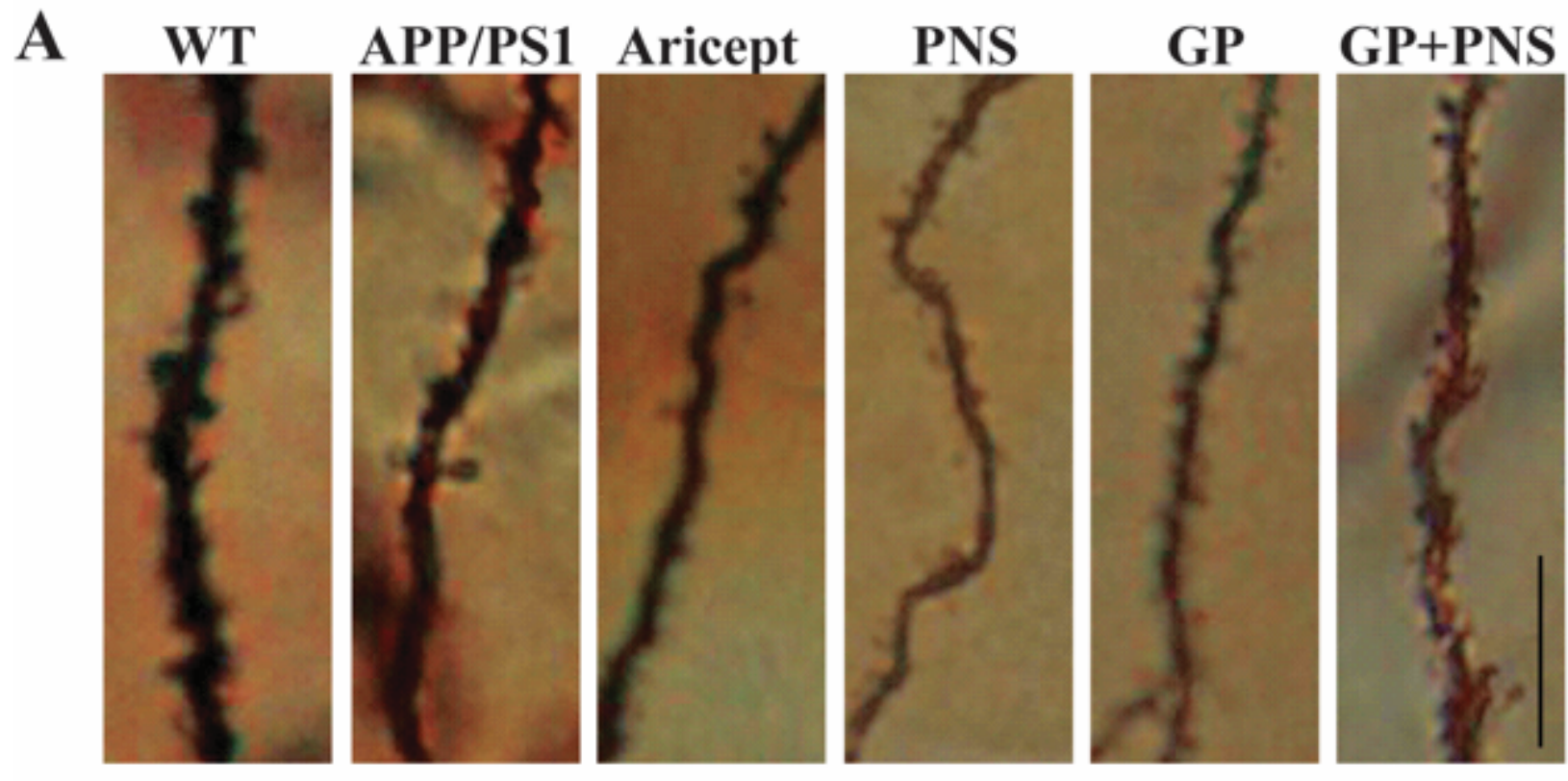

B

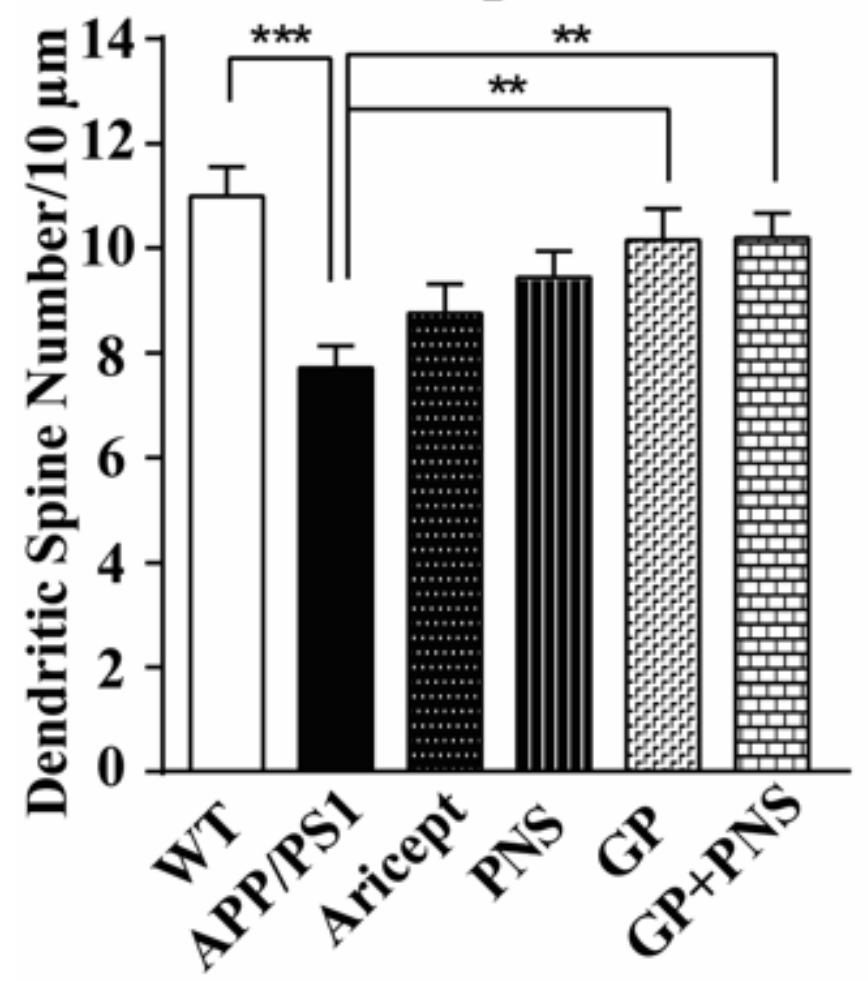

Apical
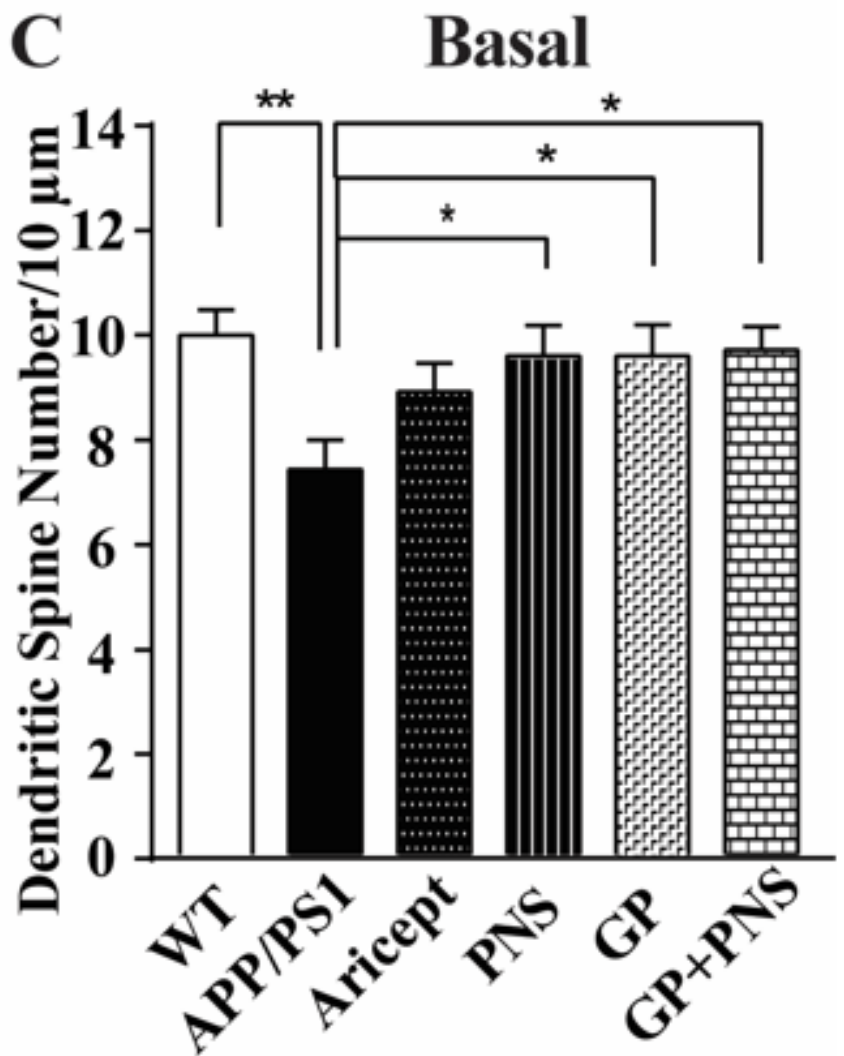

Figure 3 
TLJN increases the number of dendritic spines at hippocampus CA1 areas in APP/PS1 Tg mice. (A).A typical figure to illustrate dendritic spines in hippocampus CA1 area are by Golgi silver staining. At 4month old age, in WT mice, its dendritic spines in hippocampus CA1 area are evenly dispersed with a high density. Identical to WT, in PNS+GP group, again, it shows a high density and evenly dispersed. However, unlikely to WT, in Tg and Aricept groups, their dendritic spines occurred sporadically uneven with low density. Scale bar: $10 \mu \mathrm{m}$. (B).Due to analysis the number of dendritic spines in hippocampus CA1 area, its number has been counted in both apical and basal ends. In apical ends, compared with WT, the number of dendrite spines is significantly decreased in Tg mice. After treatment, GP and PNS+GP groups significantly increase the number of dendrite spines compared with Tg. Aricept does not change the number of dendritic spines in the apical end. $(n=4)$ Data are represented as mean $\pm S E M$. $* \star * p<0.001$, ** $p<0.01$.(C).In basal ends, compared with WT, the number of dendrite spines is significantly decreased in Tg mice. After treatment, PNS, GP and PNS+GP groups significantly increase the number of dendrite spines compared with Tg. Again, Aricept does not change the number of dendritic spines in the basal end. $(n=4)$ Data are represented as mean \pm SEM. ** $p<0.01, * p<0.05$.

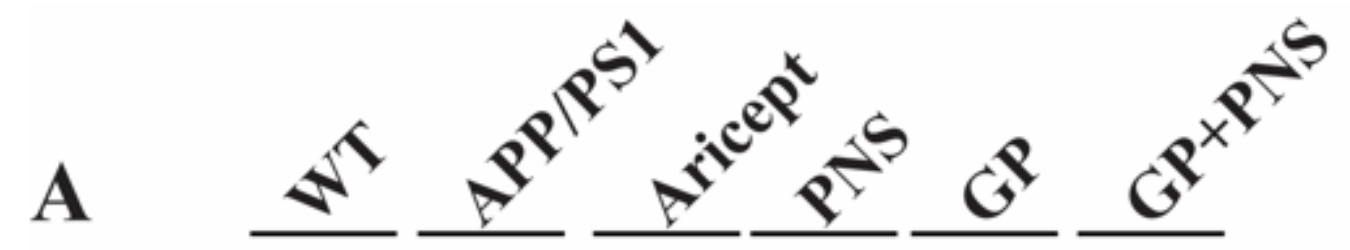

95kD-

$42 \mathrm{kD}-$
PSD-95

\section{$\beta$-actin}

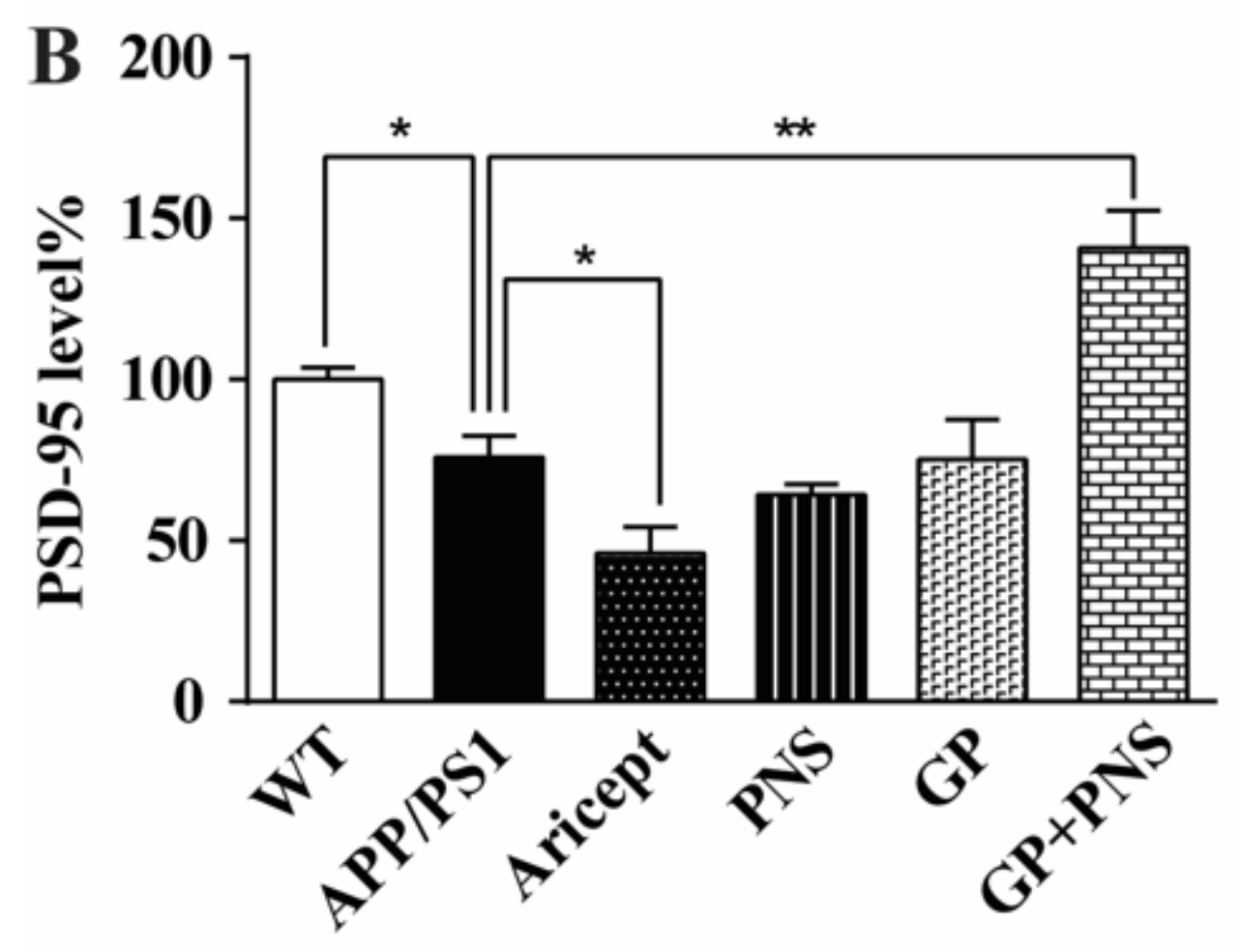


Figure 4

TLJN increases the level of synaptic function-related protein PSD-95 in APP/PS1 hippocampus. (A).A typical figure to exhibit the PSD-95 protein expression in hippocampus by Western blot. (B).At 4-month age of APP/PS1 Tg mice, its PSD-95 protein level is significantly decreased compared with WT mice. PNS+GP can strikingly increases PSD-95 protein level in APP/PS1 hippocampus. Interestingly, the protein level of PSD-95 is further reduced in Aricept group. $(n=4)$ Data are represented as mean \pm SEM. ${ }^{*} p<$ $0.01,{ }^{*} \mathrm{p}<0.05$.

\section{GP and PNS combination in early intervention}

Start at 1-month age;

Last for 3 months.

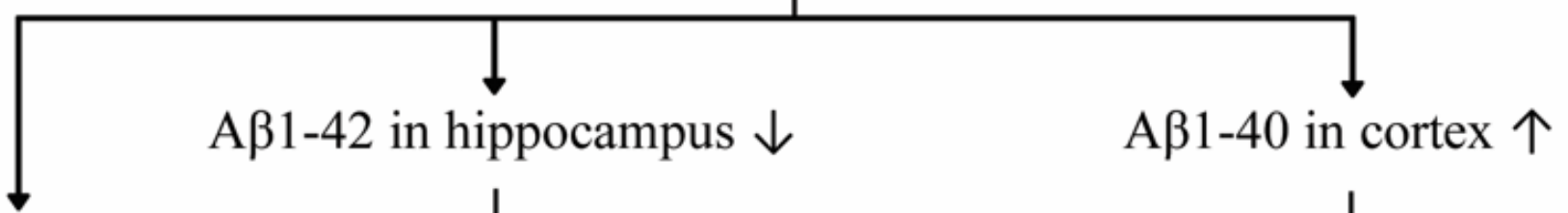

Synaptic plasticity in hippocampus $\uparrow$ : Dentritic spines $\uparrow$ PSD-95 protein level $\uparrow$

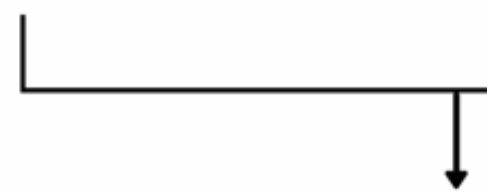

Ratios of $A \beta 1-42 / A \beta 1-40$ in both hippocampus and cortex $\downarrow$

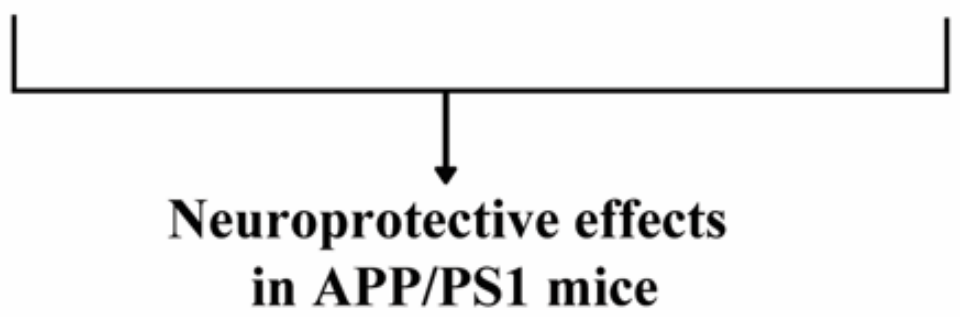

Figure 5

The neuroprotective effects of TLJN on APP/PS1 at 4-month age. 